\title{
Measurement of the neutron total cross sections of Ta and Mo and proton induced reaction cross sections of ${ }^{\text {nat }} \mathrm{Mo}$
}

\author{
Guinyun Kim ${ }^{1, a}$, A.K.M. Moinul Haque Meaze ${ }^{1}$, Mayeen Uddin Khandaker ${ }^{1}$, Manwoo Lee ${ }^{1}$, Kwangsoo Kim ${ }^{1}$, \\ Young Seok Lee ${ }^{1}$, Young Do $\mathrm{Oh}^{2}$, Hengsik Kang ${ }^{2}$, Moo-Hyun $\mathrm{Cho}^{2}$, In Soo $\mathrm{Ko}^{2}$, Won Namkung ${ }^{2}$, \\ Young-Ae $\mathrm{Kim}^{3}$, Kun Joong $\mathrm{Yoo}^{3}$, and Young-Ouk Lee ${ }^{3}$ \\ 1 Kyungpook National University, Daegu 702-701, Korea \\ 2 Pohang University of Science and Technology, Pohang 790-784, Korea \\ 3 Korea Atomic Energy Research Institute, Daejeon 305-600, Korea
}

\begin{abstract}
The Pohang Neutron Facility based on an electron linear accelerator was operated for the neutron total cross section measurements by using the pulsed neutrons produced in a water-cooled Ta-target with a water moderator. The neutron total cross sections of $\mathrm{Ta}$ and $\mathrm{Mo}$ were measured in the neutron energy region from $0.01 \mathrm{eV}$ to $100-200 \mathrm{eV}$ by using the time-of-flight method. A ${ }^{6} \mathrm{LiZnS}(\mathrm{Ag})$ glass scintillator with a diameter of $12.5 \mathrm{~cm}$ and a thickness of $1.6 \mathrm{~cm}$ was used as a neutron detector. The neutron flight path from the water-cooled Ta target to the neutron detector was $12.1 \mathrm{~m}$. The background level was determined by using notch-filters of Co, In, Ta, and Cd sheets. In order to reduce the gamma rays from Bremsstrahlung and those from neutron capture, we employed a neutron-gamma separation system based on their different pulse shapes. The present measurements of Ta and Mo are in general agreement with the evaluated data in ENDF/B-VI. Cross sections for residual radio-nuclide production by proton-induced reactions on natural molybdenum were measured from their respective threshold energies to $42 \mathrm{MeV}$ by using the MC-50 cyclotron at KIRAMS. The activation method and a stacked-foil technique using high-resolution HPGe gamma-ray spectrometry were applied to determine the excitation functions. The reactions induced on $\mathrm{Al}$ and $\mathrm{Cu}$ foils were used to monitor the parameters of the proton beam. The present results are in generally good agreement with the earlier reported data and with the calculations based on the ACILE-IPPE code.
\end{abstract}

\section{Introduction}

The Pohang Neutron Facility (PNF) was proposed in 1997 and constructed at the Pohang Accelerator Laboratory on December 1998 [1]. It consists of a 65-MeV electron linac, water-cooled Ta-target, and a 12-m-long evacuated vertical flight tube leading to the detector. The electron linac consists of a thermionic RF-gun, an alpha magnet, four quadrupole magnets, two SLAC-type accelerating sections, a quadrupole triplet, and a beam-analyzing magnet. The overall length of the linac is about $15 \mathrm{~m}$. As a photoneutron target, we choose tantalum, which has advantages of high density $\left(16.6 \mathrm{~g} / \mathrm{cm}^{3}\right)$, high melting point $\left(3,017^{\circ} \mathrm{C}\right)$, and high resistant against the corrosion by cooling water. The characteristics of PNF are described elsewhere [2].

We report the measured neutron total cross sections of natural $\mathrm{Ta}$ and $\mathrm{Mo}$ in the neutron energy range between $0.01 \mathrm{eV}$ and $100 \mathrm{eV}$ by using the neutron time-of-flight (TOF) method at the PNF. The results were compared with other measurements and the evaluated data in ENDF/B-VI.

We also report cross sections for residual radio-nuclide production by proton-induced reactions on natural molybdenum were measured from their respective threshold energy to $42 \mathrm{MeV}$ using a MC-50 cyclotron at the Korea Institute of Radiological and Medical Sciences (KIRAMS). The activation method and the stacked-foil technique using high-resolution

${ }^{a}$ Presenting author, e-mail: gnkim@knu.ac.kr
HPGe gamma-ray spectrometry were applied to determine the excitation functions.

\section{Experimental procedure}

There are two different experimental procedures: one for neutron total cross section measurements of Ta and Mo, and the other for proton induced reaction cross sections on natural Mo.

\subsection{Experiment for total cross sections}

Since the experimental method has been published previously [3-5], only a general describiton is given here. Pulsed neutrons are produced when $65-\mathrm{MeV}$ electron hit the Ta-target. The neutron guide tubes were constructed of stainless steel with two different diameters, $15 \mathrm{~cm}$ and $20 \mathrm{~cm}$, and were placed perpendicularly to the electron beam. The neutron collimation system was mainly composed of $\mathrm{H}_{3} \mathrm{BO}_{3}, \mathrm{~Pb}$, and $\mathrm{Fe}$ collimators, which were symmetrically tapered from a 10 $\mathrm{cm}$ diameter at the beginning to a $5-\mathrm{cm}$ diameter in the middle position where the sample changer was located, to an $8-\mathrm{cm}$ diameter at the end of the guide tube where the neutron detector was placed. There was a 1.8-m-thick concrete slab between the target and the detector room. The transmission samples were placed at the midpoint of the flight path and were cycled into the neutron beam by using an automatic sample changer with four sample positions. The neutron detector was 

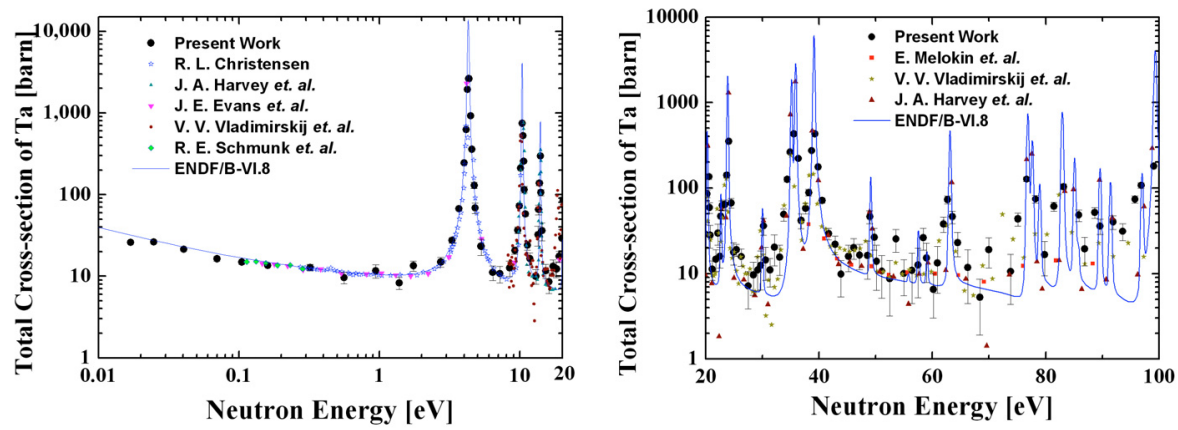

Fig. 1. Comparison of the measured total cross sections of natural Ta with other measured data and with the evaluated data in the neutron energy between $0.01 \mathrm{eV}$ and $100 \mathrm{eV}$.
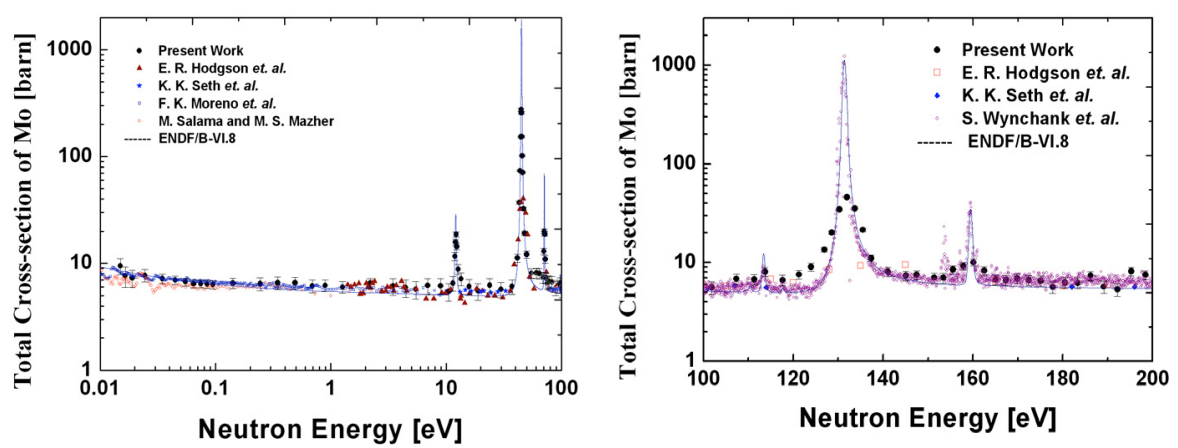

Fig. 2. Comparison of the measured total cross sections of natural Mo with other measured data and with the evaluated data in the neutron energy between $0.01 \mathrm{eV}$ and $200 \mathrm{eV}$.

located at a distance of about $12 \mathrm{~m}$ from the neutron target. A ${ }^{6} \mathrm{Li}-\mathrm{ZnS}(\mathrm{Ag})$ scintillator (BC702) with a diameter of $12.5 \mathrm{~cm}$ and a thickness of $1.6 \mathrm{~cm}$ was used as the neutron detector.

During the transmission measurement, the electron linac was operated with a repetition rate of $12 \mathrm{~Hz}$, a pulse width of $1.0 \mu \mathrm{s}$, and an electron energy of $65 \mathrm{MeV}$. The measurements were performed with two samples and two open positions in the sample changer. The positions of the samples were chosen in the following sequence: sample 1, open, sample 2, and open. The thickness of the Ta and Mo samples are $0.45 \mathrm{~mm}$ and $1.5 \mathrm{~mm}$, respectively. In addition to these samples, a set of notch filters of Co, In, and Cd plates, $0.5 \mathrm{~mm}$, $0.2 \mathrm{~mm}$, and $0.5 \mathrm{~mm}$ in thickness, respectively, was used for the background measurement and the energy calibration. The exposure time for each sample position was 15 minutes; for each open position, it was 7.5 minutes. The total data taking times for the Ta and the Mo samples were 45 and 20.5 hours, respectively; the time was the same for the open beam.

\subsection{Experiment for proton-induced reaction}

The cross sections of the proton-induced reactions on natural molybdenum were measured as a function of the proton energy in the range from threshold energy to $40 \mathrm{MeV}$ by using a conventional stacked-foil activation technique combined with high-resolution gamma-ray spectrometry. The $\mathrm{Al}$ and the $\mathrm{Cu}$ foils were used to monitor the beam intensity and to degrade the beam energy, respectively. Three different stacks of natural Mo with monitor foils were irradiated by $27.5 \mathrm{MeV}, 31.4 \mathrm{MeV}$, and $42.1 \mathrm{MeV}$ collimated proton beam with a diameter of $10 \mathrm{~mm}$, and a beam current of about $50 \mathrm{nA}$ in the MC-50 cyclotron.

After the irradiations and appropriate cooling time, the samples were taken off, and the induced gamma activities emitted from the activation foils were measured by using a HPGe detector with a diameter of $55.1 \mathrm{~mm}$ and a thickness of $52.2 \mathrm{~mm}$. The energy resolution of the detector was $1.90 \mathrm{keV}$ full width at half maximum at the $1332.5-\mathrm{keV}$ peak of ${ }^{60} \mathrm{Co}$. The photopeak efficiency curve of the gamma spectrometer was calibrated with a set of standard sources. The proton beam energy degradation along the stacked foils was determined using the computer program SRIM-2003 [6]. The proton beam intensity was determined by using the monitor reactions, ${ }^{27} \mathrm{Al}(\mathrm{p}, \mathrm{x}){ }^{24} \mathrm{Na}$ and ${ }^{\text {nat }} \mathrm{Cu}(\mathrm{p}, \mathrm{x}){ }^{62} \mathrm{Zn}$ from the measured activities induced in monitor foils at the front position of each stack considering that the monitor foils were irradiated simultaneously and measured in the same counting geometry.

\section{Data analysis and results}

\subsection{Total cross sections of Ta and Mo}

The total cross section is determined by measuring the transmission of neutrons through the sample. The transmission rate 
of neutrons is defined as the fraction of incident neutrons passing through the sample compared to that in the open beam. The background level was estimated by using the resonance energies of the neutron TOF spectra of notch filters of Co, In, and $\mathrm{Cd}$.

The total cross sections of natural Ta and Mo were obtained in the neutron energy range from 0.01 to $100 \mathrm{eV}$. The overall statistical errors for the measured total cross sections are less than 5\%. The systematic uncertainties came from the following sources: uncertainties from a) the flight path measurement $(\sim 2 \%)$, b) the background estimate $(<0.04 \%)$, and c) the dead time, the normalization, etc. $(<2 \%)$. Thus, total systematic error of the present measurement is less than $3 \%$. The present measurement for Ta in the neutron energy region from $0.01 \mathrm{eV}$ to $100 \mathrm{eV}$ was generally in good agreement with other data [7] and with the evaluated data in ENDF/B-VI.8 [8], as shown in figure 1 .

The measured total cross sections of natural Mo in the neutron energy region from $0.01 \mathrm{eV}$ to $200 \mathrm{eV}$ are generally in good agreement with other data [9] and with the evaluated data in ENDF/B-VI.8 [8], as shown in figure 2.

\subsection{Proton-induced reaction cross sections}

The cross sections for the reactions ${ }^{\text {nat }} \mathrm{Mo}(\mathrm{p}, \mathrm{xn})$ were determined in the proton energy range from $2.5 \mathrm{MeV}$ to $40 \mathrm{MeV}$ by using the well-known activation formula. The decay data for the monitors and Mo were taken from the table of radioactive isotopes [10]. The threshold energies were taken from the T-2 nuclear information service on the internet [11]. The standard cross sections for the monitor reactions were taken from the internet service [12]. The total uncertainties were derived considering the following: the statistical error ( $1 \sim 13 \%)$, which mainly depended on the gamma peak areas due to the different beam energies in the sample, the error in the monitor flux $(2 \sim 4 \%)$, the error due to the beam flux energy $(2 \sim 4 \%)$, the error due to the detector efficiency $(0.5 \sim 2 \%)$, and the error due to the gamma-ray intensity $(1 \sim 2 \%)$. The overall uncertainty of the cross section measurement was around $10 \%$.

The cross section for ${ }^{100} \mathrm{Mo}(\mathrm{p}, 2 \mathrm{n}){ }^{99 \mathrm{~m}} \mathrm{Tc}$ reaction is determined through the analysis of a $140.5 \mathrm{keV} \gamma$-ray peak. The ${ }^{99 \mathrm{~m}} \mathrm{Tc}$ can be produced in two processes. One is direct process through the reaction ${ }^{100} \mathrm{Mo}(\mathrm{p}, 2 \mathrm{n}){ }^{99 \mathrm{~m}} \mathrm{Tc}$ and the other is indirect process via ${ }^{100} \mathrm{Mo}(\mathrm{p}, \mathrm{pn}){ }^{99} \mathrm{Mo} \rightarrow{ }^{99 m} \mathrm{Tc}$ reaction. The present result of ${ }^{99 \mathrm{~m}} \mathrm{Tc}$ radionuclide formation is obtained via measurements on ${ }^{\text {nat }} \mathrm{Mo}$ and extrapolation of the results to $97.4 \%$ enrichment of ${ }^{100} \mathrm{Mo}$ and is shown in figure 3 in comparison with the results of other authors. Our data is in general good agreement with the data reported by Scholten et al. [13], where they used $97.4 \%$ enrichment of ${ }^{100} \mathrm{Mo}$, and those of Takacs et al. [14]. The data reported by Levkovskji [15] agree with our data in the energy range from 20 to $30 \mathrm{MeV}$ but are about $50 \%$ higher in the region of the maximum. Our previous data [16] were consistent with the present data except one point around $17 \mathrm{MeV}$. The results of Lagunas-Solar et al. [17] differ considerably from our data up to about $30 \mathrm{MeV}$ and are in complete disagreement with other data above $30 \mathrm{MeV}$, where it is difficult to separate the contributions from ${ }^{99} \mathrm{Mo}$ and ${ }^{90} \mathrm{Nb}$ radionuclides at the $140.5 \mathrm{keV} \gamma$-ray peak.

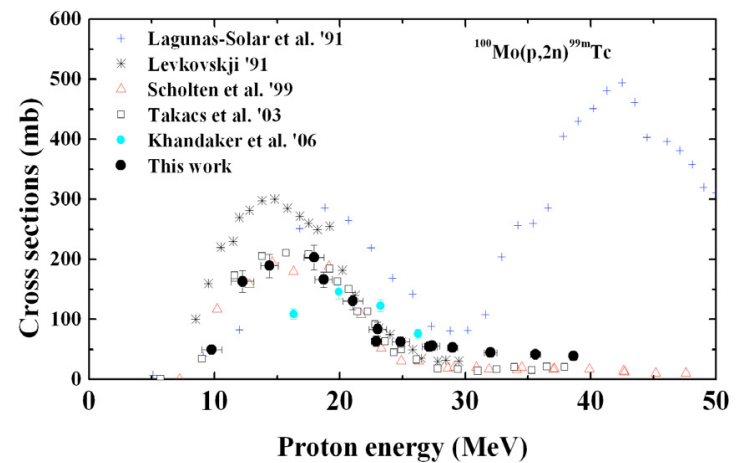

Fig. 3. Comparison of the measured cross section for ${ }^{99 \mathrm{~m}} \mathrm{Tc}$ radio nuclide formation with other measured data.

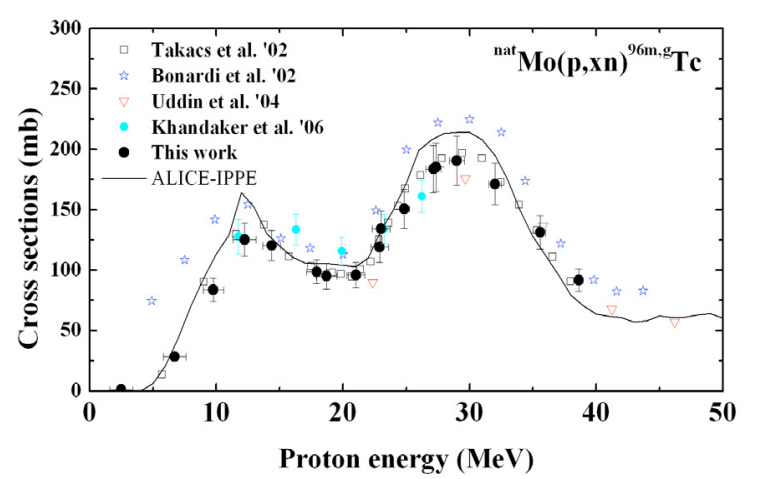

Fig. 4. Comparison of the measured cross section for ${ }^{96 \mathrm{~m}, \mathrm{~g}} \mathrm{Tc}$ radionuclide formation with other measured data.

We measured the production cross sections of ${ }^{96 \mathrm{~m}, \mathrm{~g}} \mathrm{Tc}$ radionuclide with the analysis of $778.2 \mathrm{keV} \gamma$-ray peak because both ${ }^{96 \mathrm{~m}} \mathrm{Tc}$ and ${ }^{96 \mathrm{~g}} \mathrm{Tc}$ radionuclides have contribution at $778.2 \mathrm{keV} \gamma$-ray peak. We confirmed consistency between the results of $812.5 \mathrm{keV}$ and $778.2 \mathrm{keV} \gamma$-rays. The production cross sections obtained from the $812.5 \mathrm{keV} \gamma$-ray peak are about $1 \sim 2 \%$ lower than those from the $778.2 \mathrm{keV} \gamma$-ray peak, that is reasonable because the $812.5 \mathrm{keV} \gamma$-line is the intense independent $\gamma$-ray only from a ${ }^{96 \mathrm{~g}} \mathrm{Tc}$. The measured production cross sections of ${ }^{96 \mathrm{~m}, \mathrm{~g}} \mathrm{Tc}$ are shown in figure 4 in comparison with the results of other authors. Our data showed in general good agreement with the data reported by Takacs et al. [18], Uddin et al. [19], and the theoretical data taken from ALICE-IPPE code [20]. The data reported by Bonardi et al. [21] by using natural Mo target are higher than other data.

The production cross section of ${ }^{95 \mathrm{~m}} \mathrm{Tc}$ was calculated with the analysis of the $835.1 \mathrm{keV} \gamma$-ray peak, and is shown in figure 5 compared with other reported data include our previous data [16]. Our data are in general good agreement with the recent data reported by Bonardi et al. [21] and Uddin et al. [19].

The production cross sections of the ${ }^{95 \mathrm{~g}} \mathrm{Tc}$ radionuclide were calculated using the intense independent $\gamma$-line $1073.7 \mathrm{keV}$, and confirmed the result with the $765.8 \mathrm{keV}$ $\gamma$-line. The present result of ${ }^{95 \mathrm{~g}} \mathrm{Tc}$ radionuclide formation is shown in figure 6 . The present results showed good agreement with the data reported by Bonardi et al. [21] and by Uddin et al. 


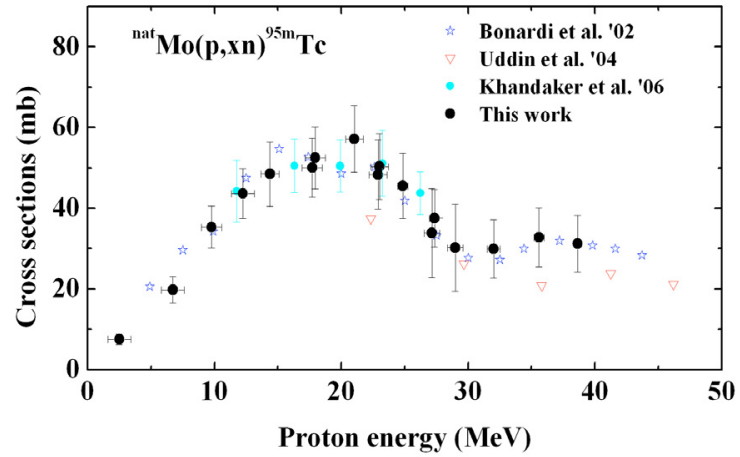

Fig. 5. Comparison of the measured cross section for ${ }^{95 \mathrm{~m}} \mathrm{Tc}$ radionuclide formation with other measured data.

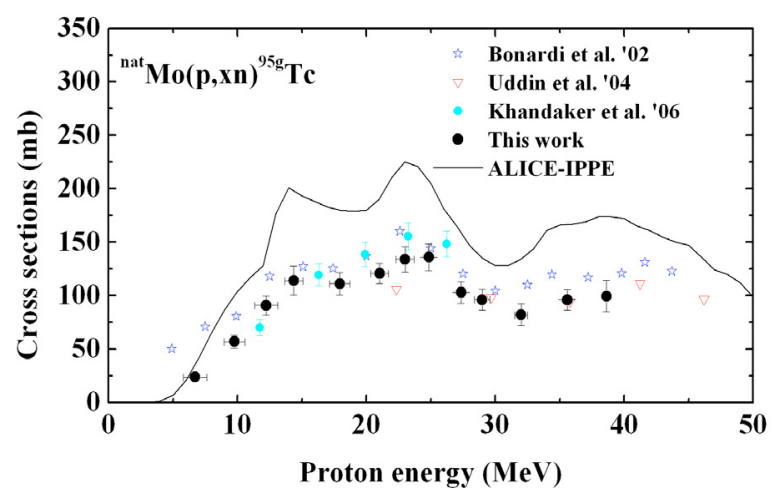

Fig. 6. Comparison of the measured cross section for ${ }^{95 \mathrm{~g}} \mathrm{Tc}$ radionuclide formation with other measured data.

[19]. The trend of peak formation agrees with the theoretical data taken from ACILE-IPPE code [20].

\section{Summary and conclusion}

The total cross sections of natural tantalum and molybdenum were measured at the Pohang Neutron Facility in the neutron energy region from $0.01 \mathrm{eV}$ to $100-200 \mathrm{eV}$ by using the TOF method and a ${ }^{6} \mathrm{Li}-\mathrm{ZnS}(\mathrm{Ag})$ glass scintillator as a neutron detector. The present measurement is in good agreement with other experimental data as well as the evaluated data in ENDF/B-VI.8.

We measured the production cross sections of ${ }^{99 \mathrm{~m}} \mathrm{Tc}$, ${ }^{96 \mathrm{~m}, \mathrm{~g}} \mathrm{Tc},{ }^{95 \mathrm{~m}} \mathrm{Tc}$, and ${ }^{95 \mathrm{~g}} \mathrm{Tc}$ radio nuclides with an overall uncertainty of about $12 \%$ for the proton-induced reactions on natural molybdenum in the energy range of $2.5-40 \mathrm{MeV}$ by using a stacked-foil activation technique.

The authors would like to express their sincere thanks to the staff of the Pohang Accelerator Laboratory and the MC-50 Cyclotron Laboratory in KIRAMS. This work was partly supported by the Korean Science and Engineering Foundation, by the Long-term Nuclear R\&D Program of the Korean Atomic Energy Research Institute, and by the BK21 program of the Korea Research Foundation.

\section{References}

1. G.N. Kim et al., in Proceedings of the International Conference on Nuclear Data for Science and Technology, 1997, edited by G. Reffo, A. Ventura, C. Grandi (Trieste, 1997), p. 556; H.S. Kang et al., in Proceedings of the First Asian Particle Accelerator Conference, 1998, edited by Y.H. Chin (Tsukuba, 1998), p. 743.

2. G.N. Kim et al., J. Kor. Phys. Soc. 38, 14 (2001).

3. A.K.M.M.H. Meaze et al., J. Kor. Phys. Soc. 46, 401 (2005).

4. G.N. Kim et al., J. Kor. Phys. Soc. 43, 479 (2003).

5. A.K.M.M.H. Meaze et al., J. Kor. Phys. Soc. 48, 827 (2006).

6. J.F. Ziegler, J.P. Biersack, U. Littmark, SRIM 2003 Code, Version 96.xx, The Stopping and Range of Ions in Solids (Pergamon, New York, 2003).

7. E. Melkonian, W.W. Havens Jr, L.J. Rainwater, Phys. Rev. 92, 702 (1953); R.L. Christensen, Phys. Rev. 92, 1509 (1953); J.A. Harvey, D.J. Hughes, R.S. Carter, V.E. Pilcher, Phys. Rev. 99, 10 (1955); J.E. Evans, E.G. Joki, R.R. Smith, Phys. Rev. 97, 565 (1955); R.E. Schmunk, P.D. Randolf, R.M. Brugger, Nucl. Sci. Eng. 7, 193 (1960); V.V. Vladimirskij et al., in http://www.nndc.bnl.gov/exfor/, EXFOR No. 40380.007.

8. M.B. Chadwick et al., Evaluated Nuclear Data File, ENDF/BVI.8, in http://www-nds.iaea.org/exfor/endf00.htm (2005).

9. J.R. Dunning, G.B. Pegrem, G.A. Pink, D.P. Mitchell, Phys. Rev. 48, 265 (1935); S. Wynchank, J. Garg, W. Havens, J. Rainwater, Phys. Rev. 166, 1234 (1968); E.R. Hodgson, J.F. Gallangher, E.M. Bowley, in Proceedings of the Physical Society, 1952 (London, 1952) 65A, p. 992; K.K. Seth, D.J. Hughes, R.L. Zimmerman, R.C. Garth, Phys. Rev. 110, 692 (1952); F.K. Moreno, J.R. Granada, R.E. Mayer, L.A. Remez, Atomkernenergie 31, 42 (1978); M. Salama, M.S. Mazher, Atomkernenergie 45, 282 (1984).

10. E. Browne, R.B. Firestone, in Table of Radioactive Isotopes, edited by V.S. Shirley (Wiley, New York, 986).

11. Reaction Q-values and thresholds, Los Alamos National Laboratory, T-2 Nuclear Information Service, in http://t2.lanl.gov/data/qtool.html.

12. Monitor cross section data, in http://www-nds.iaea.org/medical.

13. B. Scholten, R.M. Lambrecht, M. Cogneau, H. Vera Ruiz, Appl. Radiat. Isot. 51, 69 (1999).

14. S. Takacs, Z. Szucs, F. Tarkanyi, A. Hermanne, M. Sonck, J. Radioanal. Nucl. Chem. 257, 195 (2003).

15. V.N. Levkovskij, Middle Mass Nuclides $(A=40-100)$ Activation Cross-sections by Medium Energy $(E=10-50 \mathrm{MeV})$ Protons and Alpha Particles (Experiment and Systematics) (Inter-vesi, Moscow, 1991).

16. M.U. Khandaker, A.K.M.M.H. Meaze, K. Kim, D. Son, G.N. Kim, Y.S. Lee, J. Kor. Phys. Soc. 48, 821 (2006).

17. M.C. Lagunas-solar, P.M. Kiefer, O.F. Carvacho, C.A. Lagunas, Y.P. Cha, Appl. Radiat. Isot. 42, 643 (1991).

18. S. Takacs, F. Tarkanyi, M. Sonck, A. Hermanne, Nucl. Instrum. Meth. B 198, 183 (2002).

19. M.S. Uddin, M. Hagiwara, F. Tarkanyi, F. Ditroi, M. Baba, Appl. Radiat. Isot. 60, 911 (2004).

20. A.I. Dityuk, A.Yu. Konobeev, V.P. Lunev, Yu.N. Shubin, New Advanced Version of Computer Code ALICE-IPPE, INDC (CCP)-410 (IAEA, Vienna, 1998); Medium Energy Activation Cross Sections data: MENDL-2P, in http://www-nds.iaea.org/nucmed.html.

21. M. Bonardi, C. Birattari, F. Groppi, E. Sabbioni, Appl. Radiat. Isot. 57, 617 (2002). 